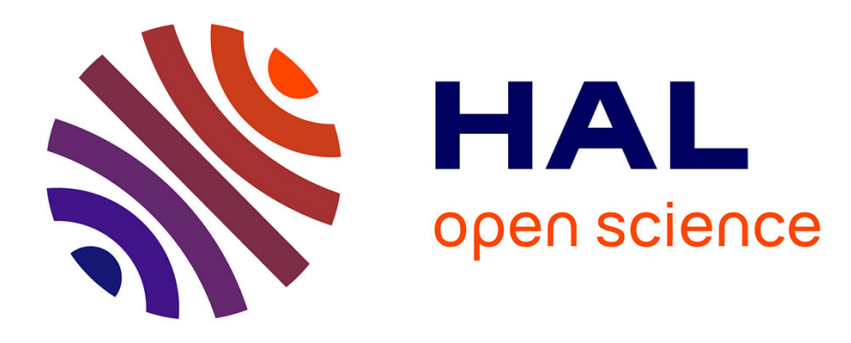

\title{
Unexpected light behaviour in periodic segmented waveguides
}

Pierre Aschieri, Valérie Doya

\section{To cite this version:}

Pierre Aschieri, Valérie Doya. Unexpected light behaviour in periodic segmented waveguides. Chaos: An Interdisciplinary Journal of Nonlinear Science, 2011, 21, pp.043118. 10.1063/1.3657377 . hal00647575

\section{HAL Id: hal-00647575 \\ https://hal.science/hal-00647575}

Submitted on 2 Dec 2011

HAL is a multi-disciplinary open access archive for the deposit and dissemination of scientific research documents, whether they are published or not. The documents may come from teaching and research institutions in France or abroad, or from public or private research centers.
L'archive ouverte pluridisciplinaire HAL, est destinée au dépôt et à la diffusion de documents scientifiques de niveau recherche, publiés ou non, émanant des établissements d'enseignement et de recherche français ou étrangers, des laboratoires publics ou privés. 


\title{
Unexpected light behaviour in periodic segmented waveguides
}

\author{
Pierre Aschiéria) and Valérie Doya \\ Laboratoire de physique de la matière condensée, CNRS: UMR6622, Université de Nice Sophia-Antipolis, France
}

(Received 11 July 2011; accepted 11 October 2011; published online 7 November 2011)

\begin{abstract}
In this article, it is shown that multimode periodic segmented waveguides (PSW) are versatile optical systems in which properties of wave chaos can be highlighted. Numerical wave analysis reveals that structures of quantum phase space of PSW are similar to Poincaré sections which display a mixed phase space where stability islands are surrounded by a chaotic sea. Then, unexpected light behavior can occur such as, input gaussian beams do not diverge during the propagation in a highly multimode waveguide. (c) 2011 American Institute of Physics.
\end{abstract} [doi:10.1063/1.3657377]

\begin{abstract}
When light is coupled into an uniform multimode waveguide, a speckle pattern is formed at the waveguide output because light travels in many different modes. If a longitudinal periodic index segmentation (or modulation) is added to the waveguide, it leads to wave chaos that strongly modify light distribution in the waveguide. Resonances appear in the quantum phase space in which the light is trapped, the modal dispersion is then canceled and the size of the input beam remains constant (i.e., collimated) all along the propagation in the waveguide.
\end{abstract}

\section{INTRODUCTION}

Optics is a privileged field to explore properties of wave chaos, and significant work has been carried out in different optical systems. Extensive investigations have been performed mostly on chaotic optical cavities and the possibility of an highly anisotropic laser emission has been highlighted for specific configurations. ${ }^{1-10}$ In D shaped fibers an optimal pump-power absorption in power amplifier has been demonstrated $^{11}$ and scars modes have also been observed. ${ }^{12}$ Other configurations such as corrugated waveguides ${ }^{13,14}$ seem to be promising systems. The purpose of the article is to show that periodic segmented waveguides (PSW) are guiding structures in which optical wave chaos can be studied and used. PSW are standard component in integrated optics domain. They have been used in linear applications to make tapers or mode filters. ${ }^{15-18}$ In nonlinear optics domain, they have been used to achieve efficient nonlinear guided wave interactions using the quasi phase matching (QPM) or balanced phase matching (BPM) schemes ${ }^{19}$ and they have also been proposed as an adjustable optical power limiter devices. ${ }^{20}$ Previous works based on ray analysis on PSW have shown an emergence of a genuine chaotic behavior of the ray dynamics ${ }^{21}$ characterized by a mixed phase space which strongly modify the transit time of rays (i.e., the ray dispersion) in the waveguide. ${ }^{22}$ Numerical wave propagation with the help of the Husimi representation, ${ }^{23}$ which is a quantum equivalent of the Poincaré section, is performed in this arti-

\footnotetext{
a)Electronic mail: pierre.aschieri@unice.fr.
}

cle and show similarities with the classical ray approach. In particular, it is shown that an incident beam may either remains collimated all along the waveguide or dispersed on many waveguide modes depending if its trajectory is constructed or not on a resonance of the Poincaré section.

The paper is organized as follows. In Sec. II, PSW are described, in Sec. III, results regarding ray analysis are given, in Sec. IV, numerical wave analysis is performed and finally, in Sec. V, perspectives and conclusions are drawn.

\section{DEVICE DESCRIPTION}

A typical PSW is sketched in Figure 1, it is formed by an array of high index segments embedded in a low index substrate. In integrated optics domain mentioned previously, PSW are mostly used in a single mode configuration but highly multimode structures are required to generate wave chaos. In order to fully satisfy this condition, the width $w$ of the waveguide is increased such that more than 70 modes are supported by the waveguide. The shape of the transverse index profile of high index segments plays a key role in the ray dynamics and previous work has shown that it has to be non-harmonic in order to generate complex ray behavior. ${ }^{21}$ A gaussian index profile is then used for high index segments which is naturally encountered with the proton exchange technique waveguide fabrication on Lithium Niobate $\left(\mathrm{LiNbO}_{3}\right)$ substrate. ${ }^{24}$ The index profile of the high index segments is given by

$$
n(x)=n_{2}+\delta n e^{-\frac{x^{2}}{w^{2}}}
$$

where $w$ is the width of the waveguide, $\delta n=n_{1}-n_{2}, n_{2}$ is the substrate index and $n_{1}$ is the maximum index induced by the waveguide fabrication process. In low index segments, the index profile is constant $n(x)=n_{2}$. The duty-cycle $(D C)$ of a PSW is commonly defined as the ratio of the length $d$ of the high index segment over the segmentation period $\Lambda$, $D C=d / \Lambda$. A step index profile is assumed along the propagation direction $z$ as it has been done in previous works $^{21,25,26}$ (a smoother profile will not change qualitatively the results).

Despite the fact that numerical values chosen to demonstrate the interest of PSW are related to proton exchanged 


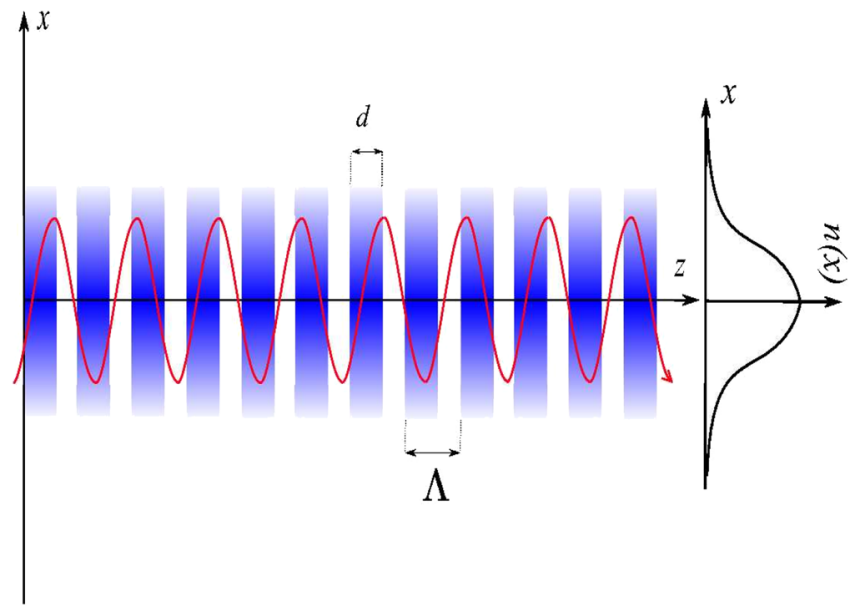

FIG. 1. (Color online) Schematic of the investigated longitudinally periodic waveguide with a period $\Lambda$ and the transverse gaussian index profile of the high index segments. Everywhere else, the index is constant and is equal to the substrate index $n_{2}$.

waveguides realized in $\mathrm{LiNbO}_{3}$, wave chaos development in PSW is not based on a specific waveguide fabrication process and may be applied to many materials.

\section{POINCARÉ SECTIONS}

Assuming that light wavelength is very small compared with the dimensions of a highly multimode waveguide, ray optics approximation can be used. A ray is defined as the path along which light energy is transmitted from one point to another in the optical system and its path is compute numerically using standard technics. ${ }^{21} \mathrm{We}$ also consider here dielectric waveguides characterized by a low index contrast $\delta n \ll 1$, which allows us to neglect reflections of rays at each interfaces. Poincaré sections are a convenient and widely used representations of a periodically perturbed nonlinear system which allow us to get a deeper insight of the ray dynamics. In the case of PSW, it consists of a periodical projection of the ray trajectory onto the phase plane.

In Figures 2(a) and 2(b), it is reported Poincaré sections for two waveguide configurations. Poincaré sections reveal the mixed aspect of the system characterized by the coexistence of a regular or a chaotic behavior of the ray trajectory depending on initial condition. Non diverging trajectories are constructed on KAM (Kolmogorov-Arnold-Moser) torus characterized by close curve circle, whereas diverging trajectories are represented by dots.
In Sec. IV, it will be performed wave analysis for same waveguide configurations.

\section{WAVE ANALYSIS}

This section reports the results of numerical simulations. The analysis has been developed using a 2D structure, and for simplicity, a TE (transverse electric) polarization has also been assumed for the fields. Using the classical assumption of the slowly envelope variation and neglecting backward propagating waves, ${ }^{15,21,25,26}$ the parabolic wave equation representing the evolution of the field in a PSW is given by

$$
2 j k_{0} n_{0} \frac{\partial E}{\partial z}-\frac{\partial^{2} E}{\partial x^{2}}-k_{0}^{2}\left(n^{2}(x, z)-n_{2}^{2}\right) E=0,
$$

where $E$ is the electric optical field, $n(x, z)=n(x, z+\Lambda)$ is the transverse waveguide profile given by Eq. (1) which is periodic in $z$ direction with a period $\Lambda, n_{0}=n_{2}+\delta n \times D C$ is used here as reference indexes, ${ }^{27} k_{0}=2 \pi / \lambda$ is the free space number for the wavelength $\lambda$. Equation (2) is then solved using standard finite difference beam propagation method (FD-BPM). ${ }^{27}$ FD-BPM provides the transverse field shape evolution along the longitudinal direction and allows to get Husimi representation. Usually Husimi distribution is defined at a particular propagation length, however, in order to compare classical and quantum phase space, a superposition of Husimi functions at periodic positions $z$ such as $z=p \Lambda$, with $p=1,2,3, \ldots$ is used. The Husimi distribution is then given by

$$
\operatorname{Hus}(x, k)=\sum_{p=1}^{N} \operatorname{Hus}(x, k, z=p \Lambda),
$$

with $k=k_{0} \sin (\theta)$.

Wave propagation has been computed for PSW configurations that have already been used in Sec. III for ray analysis, the wavelength is $\lambda=0.4 \mu \mathrm{m}$ and the width of the input gaussian beam is fixed to a value of $2.5 \mu \mathrm{m}$ for all simulations. PSW configuration corresponding to Figure 2(a) is used for wave propagation. A gaussian input filed is injected such that its parameters coincide with one of the resonance shown in the Poincaré section and it is noted "Gaussian input 1 " in Figure 2(a). The gaussian is centered at $x=-9 \mu \mathrm{m}$ and the angle of incidence is $\theta=0$. The field evolution is represented in Figure 3(a). Whereas a spreading of the input field on many modes of this mulitmode PSW is expected, the
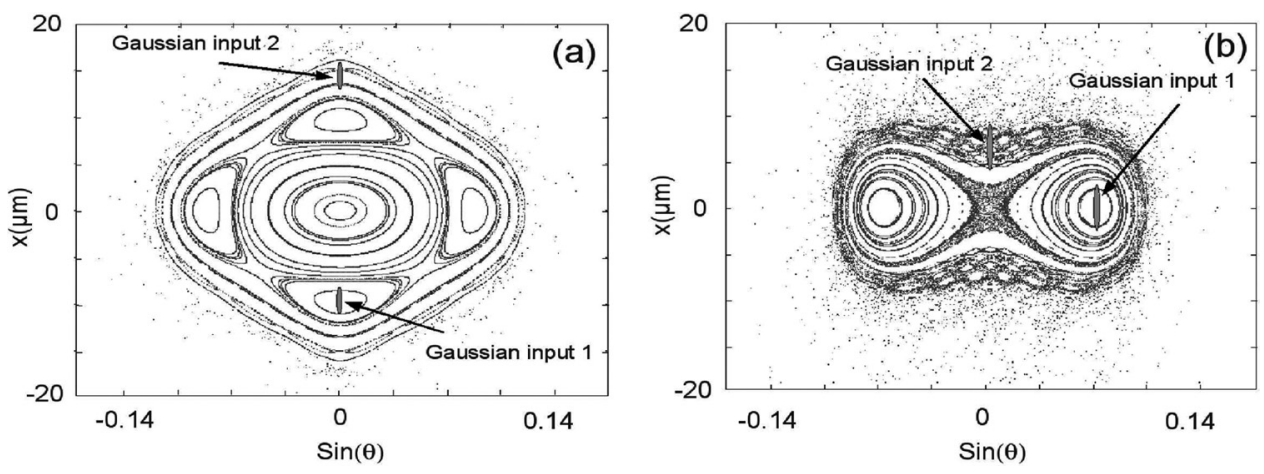

FIG. 2. Poincaré sections for two waveguide configurations. (a), $\Lambda=200 \mu \mathrm{m}$, $D C=0.8, w=15 \mu \mathrm{m}, \delta n=0.032$ and (b) $\Lambda=300 \mu \mathrm{m}, D C=0.8, w=12.75 \mu \mathrm{m}$, $\delta n=0.032$. 

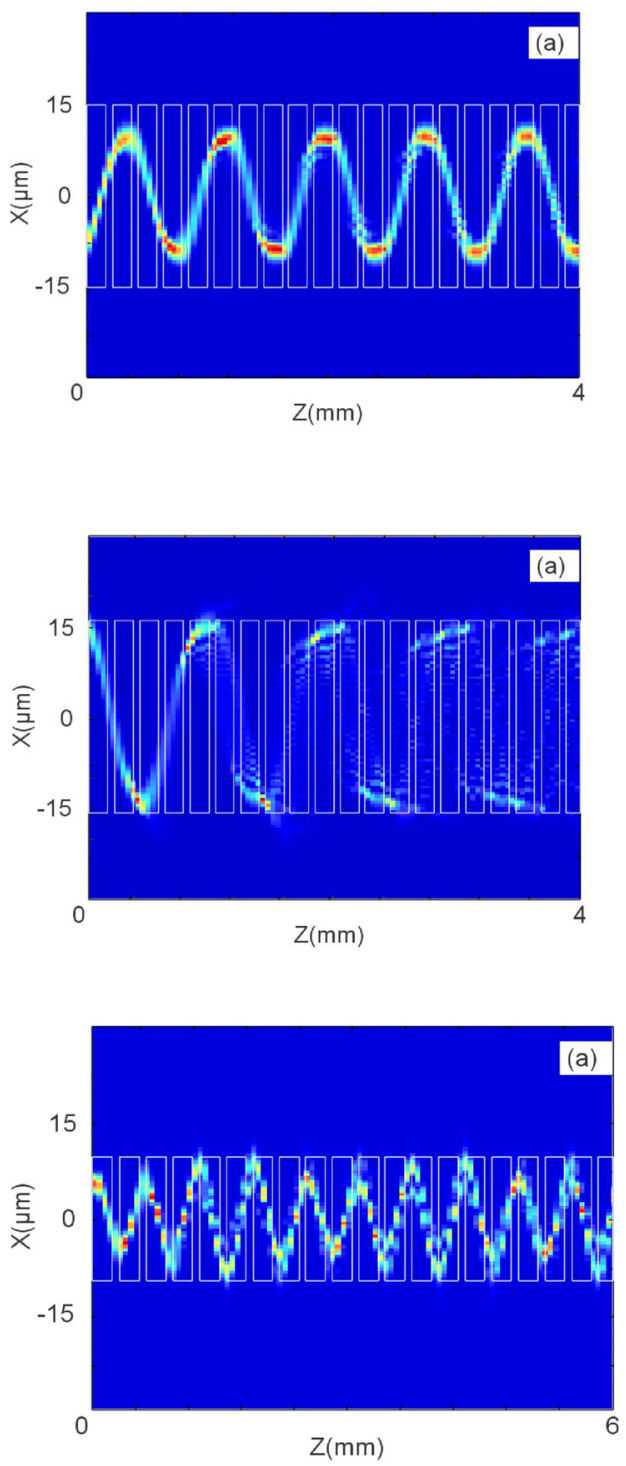

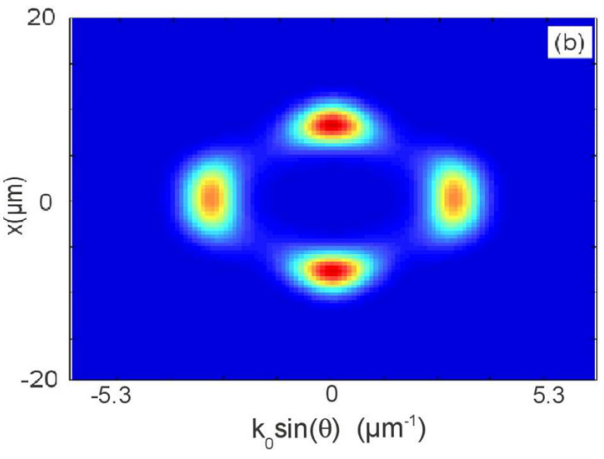

FIG. 3. (Color online) Intensity field distribution in the waveguide whose parameters are $w=15 \mu \mathrm{m}, \Lambda=200 \mu \mathrm{m}$, $D C=0.8, \delta n=0.032$ (Rectangles represent waveguide segments). The input gaussian beam is centered at $x=-9 \mu \mathrm{m}$ with an angle of incidence $\theta=0$ (a). The corresponding Husimi distribution (b)
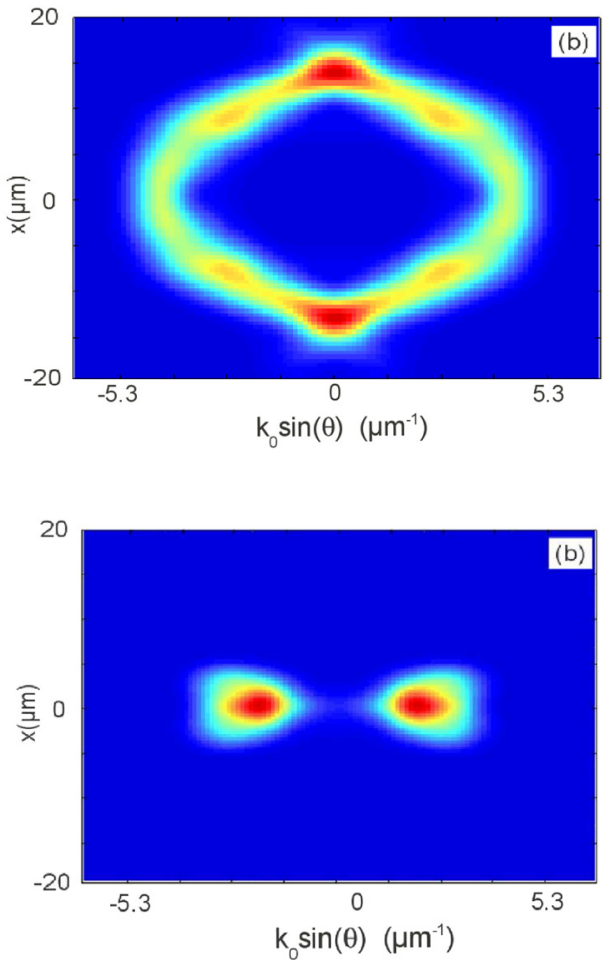

FIG. 4. (Color online) Intensity field distribution in the same waveguide as in Figure 3, the same input gaussian beam is used but shifted to $x=15 \mu \mathrm{m}$ (a). The corresponding Husimi distribution (b)

FIG. 5. (Color online) Intensity field distribution in the waveguide whose parameters are $w=12.75 \mu \mathrm{m}, \Lambda=300 \mu \mathrm{m}$, $D C=0.8, \delta n=0.032$. The input gaussian beam is centered at $x=0$ with an angle of incidence of $\theta=3.5^{\circ}$ (a). The corresponding Husimi distribution (b)

gaussian input field remains collimated and does not experience diffraction during the propagation. The corresponding Husimi distribution given in Figure 3(b) shows that there are 4 positions in the quantum phase space where the probability to find the electric field is high. These 4 spots correspond to the 4 peripheral resonances of the Poincare section. The position of the input gaussian is now modified ( $x=15 \mu \mathrm{m}$, noted "Gaussian input 2" in Figure 3(a)) such that initial conditions occur outside of resonances of the Poincaré section. Then, the input field does not remain collimated and it spreads on many waveguide modes as it is shown in Figure 4(a). The corresponding Husimi distribution (Figure 4(b)) is then constructed on a "ring" illustrating the fact that the field is present on many spatial positions and with many wave vectors. It has to be mentioned that the propagation losses are null for the two different inputs. The reason is that gaussian input parameters in both cases lie in a region of the Poincaré section which is surrounded by a densely covered circle (KAM torus). KAM torus acts as a barrier that cannot be crossed by a ray trajectory and then the light remains confined in the waveguide. For the second PSW configuration, the corresponding Poincaré section given in Figure 2(b) presents only two main resonances. The input field is firstly injected on a resonances, the gaussian beam is centered at the position $x=0$ and incident angle is $\theta=3.5^{\circ}$ (noted "Gaussian input 1 " in Figure 2(b)). The input field remains collimated all along the propagation in the waveguide and there are no propagation losses. The corresponding Husimi distribution given in Figure 5(b) exhibits two spots which correspond to resonances of the Poincaré section. If the input gaussian beam lies in the chaotic sea of the Poincaré section (at the position $x=10 \mu \mathrm{m}$ with an incident angle $\theta=0$, noted "Gaussian input 2" in Figure 2(b)), the field is then quickly dispersed in the waveguide as shown in Figure 6(a). Regarding losses, the situation has changed, the loss coefficient is $7.8 \mathrm{~dB} / \mathrm{cm}$ which corresponds to a highly dissipative guiding configuration. The difference in loss behavior is related to the structure of the Poincaré section (Figure 2(b)) the 2 resonances are surrounded by a chaotic sea and there is no peripheral KAM torus that can prevent the divergence of rays and, as a consequence, light can escape from the waveguide. The Husimi distribution given in Figure 6(b) confirms that the probability to find the optical field is high in the chaotic sea. 


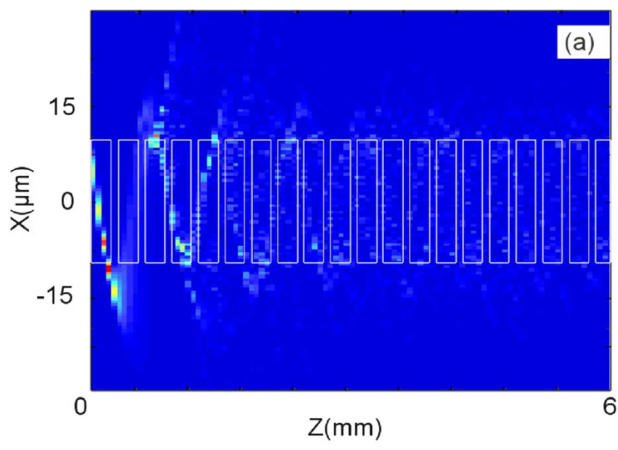

\section{CONCLUSION AND PERSPECTIVES}

The wave analysis performed in this paper confirms what was foreseen with the ray analysis that PSW are promising systems in which wave chaos could be studied and observed experimentally. The mixed aspect of the system, which was previously observed with the geometrical approach, is confirmed with the wave analysis. With an appropriate input condition for the incident beam, the presence of resonances prohibits modal dispersion and keeps the beam collimated all along a lossless propagation in the waveguide. The waveguide configuration proposed here is based on well known waveguide fabrication process on $\mathrm{LiNbO}_{3}$ substrate. It has to be recalled here that $\mathrm{LiNbO}_{3}$ is widely used in integrated optics domain because it is an excellent material for manufacture of optical waveguides but $\mathrm{LiNbO}_{3}$ also displays many properties such as electro-optic effects, $\chi^{2}$ nonlinear susceptibility and photorefractive effect which open a wide field of investigations of wave chaos in mixed phase space system. The optical refractive index could be perturbed or modified by applying an electric field via the electro-optic effect, nonlinear wave chaos could also be performed by using the photorefractive effect which is generally considered as a Kerr-like effect. Finally, thanks to the high nonlinear $\chi^{2}$ coefficient, parametric wave interactions could also be considered.

${ }^{1}$ J. U. Nockel and A. D. Stone, Nature (London) 385, 45 (1997)

${ }^{2}$ P. B. Wilkinson, T. M. Fromhold, R. P. Taylor, and A. P. Micolich, Phys. Rev. E 64, 026203 (2001).

${ }^{3}$ T. Gensty, K. Becker, I. Fischer, W. Elsäßer, C. Degen, P. Debernardi, and G. P. Bava, Phys. Rev. Lett. 94, 233901 (2005).
${ }^{4}$ J. Dingjan, E. Altewischer, M. P. van Exter, and J. P. Woerdman, Phys. Rev. Lett. 88, 064101 (2002).

${ }^{5}$ S. Shinohara and T. Harayama, Phys. Rev. E 75, 036216 (2007).

${ }^{6} \mathrm{M}$. Hentschel and K. Richter, Phys. Rev. E, 66, 056207 (2002).

${ }^{7}$ W. Fang, A. Yamilov, and H. Cao, Phys. Rev. A 72, 023815 (2005).

${ }^{8}$ S.-Y. Lee, S. Rim, J.-W. Ryu, T.-Y. Kwon, M. Choi, and C.-M. Kim, Phys. Rev. Lett. 93, 164102 (2004).

${ }^{9}$ M. Lebental, J. S. Lauret, R. Hierle, and J. Zyss, Appl. Phys. Lett. 88, 031108 (2006).

${ }^{10}$ N. Djellali, I. Gozhyk, D. Owens, S. Lozenko, M. Lebental, J. Lautru, C. Ulysse, B. Kippelen, and J. Zyss, Appl. Phys. Lett. 95, 101 (2009).

${ }^{11}$ V. Doya, O. Legrand, and F. Mortessagne, Opt. Lett. 26, 872 (2001).

${ }^{12}$ V. Doya, O. Legrand, F. Mortessagne, and C. Miniatura, Phys. Rev. Lett. 88, 014102 (2001).

${ }^{13}$ O. Bendix, J. Méndez-Bermúdez, G. Luna-Acosta, U. Kuhl, and H. J. Stökmann, Microelectron. J. 36, 285 (2005).

${ }^{14}$ E. D. Leonel, Phys. Rev. Lett. 98, 114102 (2007).

${ }^{15}$ Z. Weissman and A. Hardy, J. Lightwave Technol. 11, 1831 (1993).

${ }^{16}$ M. H. Chou, M. A. Arbore, and M. M. Fejer, Opt. Lett. 21, 794 (1996).

${ }^{17}$ D. Castaldini, P. Bassi, P. Aschiéri, S. Tascu, M. D. Micheli, and P. A. Baldi, Opt. Express 17, 17868 (2009).

${ }^{18}$ D. Castaldini, P. Bassi, S. Tascu, P. Aschieri, M. P. D. Micheli, and P. Baldi, J. Lightwave Technol. 25, 1588 (2007).

${ }^{19}$ J. D. Bierlein, D. B. Laubacher, J. B. Brown, and C. J. van der Poel, Appl. Phys. Lett. 56, 1725 (1990).

${ }^{20}$ P. Aschiéri, F. Fogli, P. Aumont, M. D. Micheli, G. Bellanca, and P. Bassi, Opt. Commun. 235, 55 (2004).

${ }^{21}$ P. Aschiéri, V. Doya, and A. Picozzi, J. Opt. A: Pure Appl. Opt. 8, 386 (2006).

${ }^{22}$ P. Aschiéri and V. Doya, Opt. Commun. 283, 3673 (2010).

${ }^{23}$ K. Husimi, Math. Soc. Jpn. 22, 264 (1940).

${ }^{24}$ M. De Micheli, J. Botineau, S. Neveu, P. Sibillot and D. B. Ostrowsky, Opt. Lett. 8, 114 (1983).

${ }^{25}$ V. Mahalakshmi, K. Thyagarajan, and M. R. Shenoy, Opt. Lett. 19, 2113 (1994).

${ }^{26}$ V. Rastogi, A. K. Ghatak, D. B. Ostrowsky, K. Thyagarajan, and M. R. Shenoy, Appl. Opt. 37, 4851 (1998).

${ }^{27}$ Y. Chung and N. Dagli, IEEE J. Quantum Electron. 26, 1335 (1990). 